\title{
The Continuing Role of Morphology in the Molecular Age
}

Juan Rosai, M.D.

National Cancer Institute, Milan, Italy

The discussion of the role that morphology plays and in all likelihood will continue to play for long time to come in "the molecular age" can be divided into two separate categories, i.e., those of diagnostic pathology and investigative pathology. Regarding the former, it is my contention that as of today there is no technique in all of medicine that provides so much information so quickly and for such little cost as the H\&E technique. Before too many eyebrows are raised, let me back up this statement with two examples. The first is a biopsy of a cervical lymph node in a 25-year-old woman that shows a papillary carcinoma featuring nuclear pseudoinclusions and psammoma bodies. The pathologist examining that section will be able to tell the clinician: "This patient has a metastatic tumor in this lymph node. The primary tumor is located in the thyroid gland, most likely in the homolateral lobe. It may be very small and not detectable by clinical or radiographic means but, trust me, it is there. It is made up of epithelial, more specifically glandular, and even more specifically thyroidal follicular cells. It is likely to be accompanied by multicentric microscopic foci throughout the gland. If radiation had been administered to the neck of this patient during infancy, you should assume a causal relationship with the tumor. Other cervical lymph nodes are likely to be involved, particularly those in the central compartment. The lung is the other site you should look at for additional metastases, but other organs are not likely to be affected at this point. These tumor cells have the capacity to incorporate iodine. This means that if there are any additional tumor foci in this patient, they are likely to show up with a small dose of radioactive iodine, and to regress if a larger dose were to be administered. Most notably, despite the presence of a malignant tumor that has spread out beyond its original site, I can confidently tell you that 10 years from now this patient will probably be

Copyright (C) 2001 by The United States and Canadian Academy of Pathology, Inc.

VOL. 14, NO. 3, P. 258, 2001 Printed in the U.S.A.

Date of acceptance: December 12, 2000.

Address reprint requests to: Juan Rosai, M.D., Dipartimento di Patologia, Istituto nazionale Tumori, Via G. Venezian 1, 20133 Milano, Italy. alive and well." The second example is a biopsy from a rapidly growing large mass centered in the thyroid gland of a 72-year-old man showing a malignant spindle cell tumor featuring numerous mitoses, extensive necrosis, and blood vessel invasion. After looking at an H\&E section of this tumor, the pathologist will be able to tell his clinical colleague: "Although this tumor looks very similar to a spindle cell sarcoma of soft tissues and it may be called such by the unwary, it is in reality an undifferentiated carcinoma with a sarcoma-like appearance. It is composed of follicular cells, but has not arisen de novo from normal cells. Rather, it represents the end-of-the-line progression of a pre-existing, welldifferentiated tumor that had been there for a long time and that, in all likelihood, was a papillary carcinoma, even if I see no trace of it at present. This anaplastic carcinoma is probably all over the thyroid, has extended beyond the gland, and is accompanied by lymph node and distant metastases. No matter what treatment you will institute, I am sorry to tell you that this patient will die of this tumor within 2 years and probably in the course of the next few months."

I hope the reader will agree that the amount of information that the examination of these samples has provided is staggering. The fact that this is the case should not be too surprising. After all, the morphologic appearance of a tumor as seen in an $H \& E$ slide represents the grand synthesis of thousands of genes working in concert and sometimes in opposition, and there is probably not a single gene that plays an important role in the neoplastic process whose expression is not manifested in one way or another in a morphologic change that can be detected by those with the training and ability to do it. Therefore, from a practical medical standpoint, the challenge of any new technique, whether molecular-based or not, is to show that it can provide information of prognostic or therapeutic significance that goes above and beyond that already provided by the standard technique. It is my impression that this does not happen as often as some people claim it does. Actually, out of the hundreds of "markers" that have been described as being 
statistically associated with a clinically important feature of solid tumors (such as survival), no more than a handful will retain their alleged predictive value once these tumors have been segregated into the appropriate categories based on morphologic parameters. That fact rarely comes across in the articles one reads on these markers. The most benevolent explanation is that the authors did not think about it, perhaps because they did not have an anatomic pathologist in the study who should have raised and evaluated the issue. A more cynical interpretation is that they have made a conscious or unconscious effort to suppress that aspect of the study from their minds and from the paper.

Viewed from this angle, morphology remains the gold standard against which any claim based on new technology needs to be measured. Gonzalez-Crussi expressed this feeling very well when stating that, "as pathologists, we subscribe to the belief that the timehonored interpretation of histopathology is preeminent in tumor diagnosis. Yes: However sophisticated and 'modern,' a novel diagnostic technique ought to be suspect if it does violence to a universally agreed upon diagnosis arrived at by more traditional means" (Am J Surg Pathol 1987; 11: 491).

From the point of view of the causes and mechanisms of tumor formation, i.e., tumor etiology and pathogenesis, morphology is also in a position to play a vital role, although unfortunately not too many people realize it. Many examples can be quoted in support of this claim - the history of desmoplastic small cell tumor being a good (and close to the heart) example. It was through morphology that the entity was first identified, a process that led to the discovery of a specific chromosomal translocation and in turn to a specific gene fusion. One wonders how long would it have taken for the molecular techniques to detect by themselves the uniqueness of the change and from there the existence of an entity. Actually, if one thinks about the major advances that have been made in the field in recent years, one realizes that most of them (at least in the field of solid tumors) have occurred as a result of the symbiosis of pathologists and molecular biologists, rather than each of them working in isolation.

The Director of the NCI issued last year a much publicized "Director Challenge" for a new classification of tumors based on molecular parameters that will replace the existing morphology-based classification and that will provide clinically more meaningful information. This high aim would greatly benefit from a slight modification to the premise. I think that the new classification will derive from a blending of the information derived from morphology and molecular biology rather than the substitution of one set of information for another. Even if one were to accept that eventually the molecular-based information will be self- sufficient, I seriously doubt whether this will occur without morphology having contributed mightily to every step of the process.

Perhaps I may be allowed to conclude this diatribe by sharing some preliminary and probably hopelessly naive conclusions I have drawn from some of the tumor molecular alterations that have been described recently.

- A clearcut correlation exists between some cytogenetic/molecular alterations and the tumor morphology/phenotypes. Well-known examples are synovial sarcoma, Ewing sarcoma/ PNET, alveolar rhabdomyosarcoma, and extraskeletal myxoid chondrosarcoma.

- This correlation sometimes also operates at the level of the microscopic subtype or variety of the tumor, as in the various forms of adipose tissue neoplasms or the two major forms of synovial sarcoma.

- The level of some of these correlations is impressive, but few if any of them reach the $100 \%$ level. Contradictory results are being documented, and it is likely that many more will be recorded in the near future. This should not come as a big surprise because it is exactly what has happened with all other techniques in pathology, including electron microscopy and immunohistochemistry.

- Correlations between specific molecular alterations and the fundamental biologic properties of the tumor (such as the capability to metastasize or the overall prognosis) are less numerous and impressive, particularly when one takes into account the factors stated in the introduction.

- It is difficult to believe that any of the single genetic alterations found in the various types of solid tumors are sufficient by themselves to confer to cells all of the behavioral properties of the tumors in which they occur. The evidence suggests instead that those alterations can be present in otherwise normal cells, while still accepting the possibility of those changes predisposing the cells that contain them to evolve into fully neoplastic elements and/or displaying the phenotypical features associated with those molecular alterations when becoming neoplastic.

- It may be premature to assume that the presence in two separate tumor nodules of one or more identical molecular alterations indicates that those two tumors arose from the same cell, and that therefore one is a metastasis from the other (or, alternatively, that both of them are metastases from a third source). It seems just as logical to assume that a given carcinogenic stimulus can induce independently the same molecular alterations in two or more cells. 
- When the genes truly responsible for the neoplastic behavior will be identified, it is likely that they will be found to cross the conventional "histogenetic" lines into which tumors have been compartmentalized. It is through this route that the new "molecular classification" of tumors asked for in the NCI Director Challenge may come to fruition.
- It is likely that the ultimate mechanism for the behavior of cancer cells will be found not to be the result of the grand total of genetic alterations but rather one or more key events that reactivate mechanisms already present in the cells, which fulfilled sometime in the past (whether ontogenetically or phylogenetically speaking) a crucial biologic task. 